\title{
Theoretical uncertainties in extracting cosmic-ray diffusion parameters: the boron-to-carbon ratio
}

\author{
Y. Genolini*, A. Putze, P. Salati and P. D. Serpico \\ LAPTh, Université Savoie Mont Blanc E CNRS, 9 Chemin de Bellevue, B.P.110 \\ Annecy-le-Vieux, F-74941, France \\ E-mail: yoann.genolini@lapth.cnrs.fr
}

PAMELA and, more recently, AMS-02, are ushering us into a new era of greatly reduced statistical uncertainties in experimental measurements of cosmic ray fluxes. In particular, new determinations of traditional diagnostic tools such as the boron to carbon ratio $(\mathrm{B} / \mathrm{C})$ are expected to significantly reduce errors on cosmic-ray diffusion parameters, with important implications for astroparticle physics, ranging from inferring primary source spectra to indirect dark matter searches. It is timely to stress, however, that the conclusions inferred crucially depend on the framework in which the data are interpreted as well as on some nuclear input parameters. We aim at assessing the theoretical uncertainties affecting the outcome, with models as simple as possible while still retaining the key dependences. We compare different semi-analytical, two-zone model descriptions of cosmic ray transport in the Galaxy: infinite slab(1D), cylindrical symmetry(2D) with homogeneous sources, cylindrical symmetry(2D) with inhomogeneous source distribution. We tested for the effect of a primary source contamination in the boron flux by parametrically altering its flux. We also tested for nuclear cross-section uncertainties.

We find that the main theoretical bias on the determination of the diffusion coefficient index $\delta$ (up to a factor two) is represented by the assumption that no injection of Boron takes place at the source. The next most important uncertainty is represented by cross-section uncertainties, which reach $\pm 20 \%$ in $\delta$. As a comparison, nuclear uncertainties are more important than the shift in the best-fit when introducing a convective wind of velocity $\lesssim 30 \mathrm{~km} / \mathrm{s}$, with respect to a pure diffusive baseline model. Perhaps surprisingly, homogeneous 1D vs. 2D performances are comparable in determining diffusion parameters. An inhomogeneous source distribution marginally alters the central value of the diffusion coefficient normalization (at the $10 \%, 1 \sigma$ level). However, the index of the diffusion coefficient $\delta$ is basically unaltered, as well as the goodness of fit. Our study suggests that, differently for instance from leptonic case, realistic modeling of the geometry of the Galaxy and of the source distribution are of minor importance to correctly reproduce $\mathrm{B} / \mathrm{C}$ data at high-energies and thus, to a large extent, for the extraction of diffusion parameters. The discussion is based on our recent publication in A\&A [1].

The 34th International Cosmic Ray Conference,

30 July- 6 August, 2015

The Hague, The Netherlands

${ }^{*}$ Speaker. 


\section{Introduction}

The abundances of nuclei in the cosmic rays (CRs) is close to the one observed in the solar system, exept for some fragile nuclei like Li, B or B, whose binding energy is weaker. Their overabundance in CRs is explained by their secondary origin i.e their production by fragmentation of heavier nuclei colliding the ISM, contrary to primaries which are mainly produced directly at the source. Measurements of secondary to primary ratio like the $\mathrm{B} / \mathrm{C}$ have long been recognized as a tool to constraint $\mathrm{CR}$ propagation parameters although confidence intervals are still wide [2].

As regard to the new precise measurements realeased by PAMELA, and expected by AMS-02, this situation demands reassessing theoretical uncertainties to make the most in extracting (astro)physical information. We revisit this issue and found that some of them were never quantified although they can be described in a very simple 1D model. In fact, we focus on determining the diffusion coefficient, which we parameterise as conventionally in the literature:

$$
D(\mathcal{R})=D_{0} \beta\left(\frac{\mathcal{R}}{\mathcal{R}_{0}=1 \mathrm{GV}}\right)^{\delta}
$$

To do so we restrain the study to the high-energy regime (kinetic energy/nucleon $\gtrsim 10 \mathrm{GeV} / \mathrm{nuc}$ ) were only diffusion and source-related effects are important. This region is actually the least subject to parameter degeneracies from other processes (such as convective winds, reacceleration, and collisional losses) and is so the most constraining for diffusion. To gauge the impact of theoretical uncertainties on a forth-coming data analysis, we compare them with statistical ones. We base our analyses on preliminary AMS-02 data of the B/C to deal with a realistic level of statistical errors ratio [3]. In the following we first recall a simple 1D diffusion model providing our benchmark for this analysis. The simplicity of this fully analytical approach which provides a realistic description of the high-energy data let us describe the theoretical degeneracies we found: the possible injection of boron nuclei at the sources, the cross-section uncertainties, the choice of the geometry of the diffusion volume, the source distribution, or the presence of convective winds.

\section{The 1D-model framework}

The simplest approach to model the transport of cosmic-ray nuclei inside the Galaxy is to assume that their production is confined inside an infinite plane of thickness $2 h$, that is sandwiched inside an infinite diffusion volume of thickness $2 \mathrm{H}$, symmetric above and below the plane. The former region stands for the Galactic disk, which comprises the gas and the massive stars of the Milky Way, whereas the latter domain represents its magnetic halo. A sketch of this model is given on the left panel of Fig. 1. Our focus on energies above $10 \mathrm{GeV} /$ nuc allows us to neglect safely continuous energy losses, electronic capture, and reacceleration.

The well-known propagation equation for the phase space density $\psi_{a}$ of a stable nucleus $a$, with charge (atomic number) $Z_{a}$, expressed in units of particles $\mathrm{cm}^{-3}(\mathrm{GeV} / \mathrm{nuc})^{-1}$, takes the form

$$
\frac{\partial \psi_{a}}{\partial t}-\frac{\partial}{\partial z}\left(D \frac{\partial \psi_{a}}{\partial z}\right)=2 h \delta(z) \cdot q_{a}+\delta(z) \sum_{Z_{b} \geqslant Z_{a}}^{Z_{\max }} \sigma_{b \rightarrow a} \cdot v \frac{\mu}{m_{\mathrm{ISM}}} \psi_{b}-\delta(z) \cdot \sigma_{a} \cdot v \frac{\mu}{m_{\mathrm{ISM}}} \psi_{a}
$$



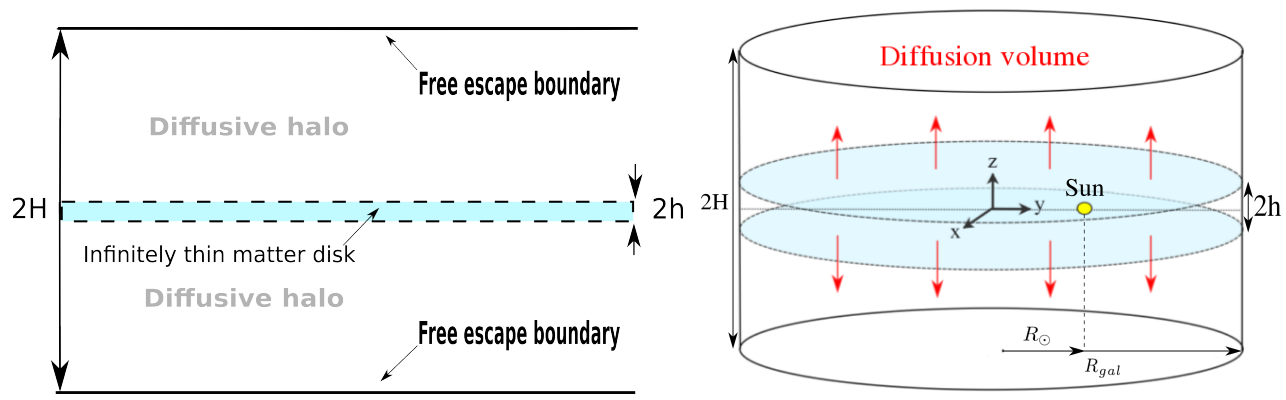

Figure 1: On the left panel : a sketch of the 1D slab model of the Galaxy, with matter homogeneously distributed inside an infinite plane of thickness $2 h$ sandwiched between two thick diffusive layers of thickness $2 H$. On the right panel, the cylindric model: the matter is homogeneously distributed inside a thin disk of thickness $2 h$ and radius $R_{g a l}=20 \mathrm{kpc}$. The solar system is at $R_{\odot} \simeq 8 \mathrm{kpc}$ from the Galactic centre.

where the spatial diffusion coefficient $D$ has been defined in Eq. (1). The cross-section for the production of the species $a$ from the species $b$ through its interactions with the interstellar medium (ISM) is denoted by $\sigma_{b \rightarrow a}$, whereas $\sigma_{a}$ is the total inelastic interaction (destruction) cross-section of the species $a$ with the ISM. The surface density of the Galactic disk is denoted by $\mu$, while $m_{\text {ISM }}$ is the average mass of the atomic gas that it contains. The values of $\sigma_{b \rightarrow a}$ and $\sigma_{a}$ are taken from the most recent formulae of [4] and [5, 6] respectively. At $10 \mathrm{GeV} /$ nuc they exhibit a plateau that allows one to approximate them as constants in this energy range. Solving the propagation Eq. (1.1) in the steady-state regime allows expressing the flux $\mathcal{J}_{a} \equiv(v / 4 \pi) \psi_{a}$ of a nucleus $a$ inside the Galactic disk $(z=0)$. However as measurements performed at Earth, in general, collect together nuclei with the same charge $Z$ irrespectively of their mass, we define the flux of one element $\mathcal{J}_{Z}$ as the sum over all its isotopes. Considering only the dominant contribution from stable nuclei, the $\mathrm{B} / \mathrm{C}$ ratio writes :

$$
\begin{aligned}
& \frac{\mathcal{J}_{\mathrm{B}}\left(E_{k}\right)}{\mathcal{J}_{\mathrm{C}}\left(E_{k}\right)}=\left\{\frac{Q_{\mathrm{B}}}{\mathcal{J}_{\mathrm{C}}}+\sigma_{\mathrm{C} \rightarrow \mathrm{B}}+\sum_{Z_{b}>Z_{\mathrm{C}}}^{Z_{\max }} \sigma_{b \rightarrow \mathrm{B}} \cdot \frac{\mathcal{J}_{b}}{\mathcal{J}_{\mathrm{C}}}\right\} /\left\{\sigma^{\mathrm{diff}}+\sigma_{\mathrm{B}}\right\} \\
& \text { where } \quad \sigma^{\mathrm{diff}}=\frac{2 D m_{\mathrm{ISM}}}{\mu \nu H} \text { and } Q_{B}=\frac{1}{4 \pi} \cdot \frac{q_{B}}{n_{\mathrm{ISM}}} \equiv N_{B}\left(\frac{\mathcal{R}}{1 \mathrm{GV}}\right)^{\alpha} .
\end{aligned}
$$

which stands for the boron source term is expressed in units of particles $(\mathrm{GeV} / \mathrm{nuc})^{-1} \mathrm{~s}^{-1} \mathrm{sr}^{-1}$. Several reasonable hypotheses are hidden behind this derivation : we assume an injetcion spectrum with the same spectral index $\alpha$ for all nuclei, and the normalisation $N_{a}$ of each isotope source term is given by the normalisation of each element $N_{Z}$ weighted by the isotopic fraction from [7].

If we assume no primary boron sources, that is, $Q_{\mathrm{B}}=0$, this expression simplifies into:

$$
\frac{\mathcal{J}_{\mathrm{B}}\left(E_{k}\right)}{\mathcal{J}_{\mathrm{C}}\left(E_{k}\right)}=\frac{\sigma_{\mathrm{C} \rightarrow \mathrm{B}}}{\sigma^{\mathrm{diff}}+\sigma_{B}}+\sum_{Z_{b}>Z_{\mathrm{C}}}^{Z_{\max }} \frac{\sigma_{b \rightarrow \mathrm{B}}}{\sigma^{\mathrm{diff}}+\sigma_{\mathrm{B}}} \cdot \frac{\mathcal{J}_{b}}{\mathcal{J}_{\mathrm{C}}} .
$$

The impact of relaxing this hypothesis is explored in the following.

The primary purpose of our analysis is to determine the diffusion parameters $D_{0}$ and $\delta$ from the $\mathrm{B} / \mathrm{C}$ flux ratio $\mathcal{F} \equiv \mathcal{J}_{\mathrm{B}} / \mathcal{J}_{\mathrm{C}}$. Basically we solve a system of triangular form(from iron to berylium), 


\begin{tabular}{lc}
\hline \hline \multicolumn{2}{c}{ Reference parameter values } \\
\hline$D_{0}\left[\mathrm{kpc}^{2} / \mathrm{Myr}\right]$ & $(5.8 \pm 0.7) \cdot 10^{-2}$ \\
$\delta$ & $0.44 \pm 0.03$ \\
$\chi_{\mathrm{B} / \mathrm{C}}^{2} /$ dof & $5.4 / 8 \approx 0.68$ \\
\hline$\gamma=\alpha-\delta$ (fixed) & -2.78 \\
\hline
\end{tabular}

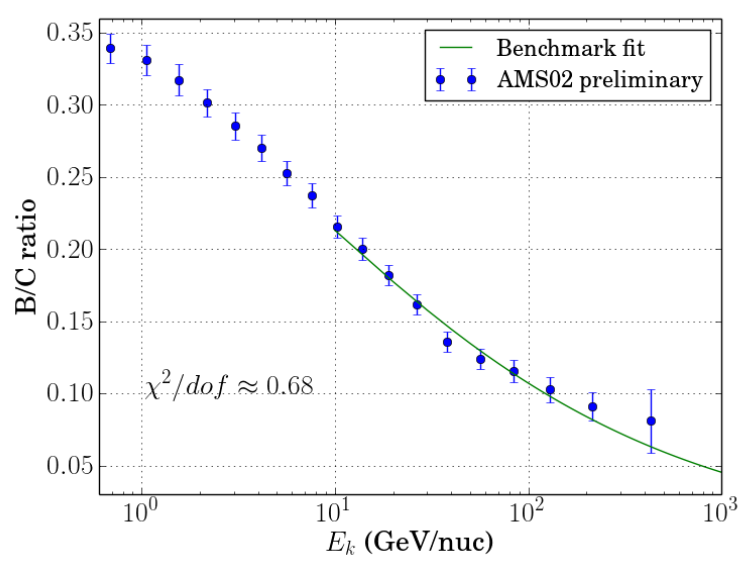

Figure 2: Benchmark best-fit parameters of the 1D/slab model, with respect to which comparisons are subsequently made. On the right panel : preliminary AMS-02 measurements of the B/C ratio [3] are plotted as a function of kinetic energy per nucleon. The theoretical prediction of the 1D/slab reference model of left-panel table is also featured for comparison.

scaling the source term thanks to nuclei fluxes borrowed from the cosmic-ray database of [8]. A minimization routine try to minimize the chi-square $\left(\chi^{2}\right)$ of the $\mathrm{B} / \mathrm{C}$ :

$$
\chi_{\mathrm{B} / \mathrm{C}}^{2}=\sum_{i}\left\{\frac{\mathcal{F}_{i}^{\exp }-\mathcal{F}_{i}^{\mathrm{th}}\left(\alpha, \delta, D_{0}, H\right)}{\sigma_{i}}\right\}^{2},
$$

As shown in Eq. (1.2), $D_{0}$ and $H$ are completely degenerate when only considering stable nuclei, which provide the bulk of cosmic rays. In the following, $H$ is therefore fixed at $4 \mathrm{kpc}$ for simplicity, although it should be kept in mind that, to a large extent, variations in $D_{0}$ can be traded for variations in $H$. Futhermore we checked that the $\mathrm{B} / \mathrm{C}$ ratio is incensitive to physical values of $\alpha$ i.e in the range [-2.5,-2]. Thus we decided to fix the value of $\gamma=\alpha-\delta$ of high-energy fluxes $\mathcal{J}_{Z}$ at Earth to the one that best fits the fluxes of the elements $Z$ that come into play in the cascade from iron to beryllium. This choice avoid to degrade the goodness of the fits on absolute fluxes in the minimization procedure. The best-fit values defining our benchmark model as well as the best-fit plot are shown in Fig. 1. As a remark we also checked that at that stage, the statistical uncertainties are still of the same order as the systematic uncertainties generated by using different energy cuts.

\section{Primary boron contamination?}

Typical fits of the $\mathrm{B} / \mathrm{C}$ ratio are based on the assumption that no boron is accelerated at the source. However, one can show easily that for typical astrophysical acceleration time and density, it leads to percent-level probabilities for nuclei to undergo spallation in the sources. A factor of only a few times higher than this would certainly have dramatic consequences on the information inferred from secondary-to-primary ratios. More elaborate versions of this idea and related phenomenology have also been detailed as a possible explanation of the hard spectrum of secondary positron data [9, 10], which was recently compared with the AMS-02 data [11]. 
In this study we challenge the ansatz $Q_{\mathrm{B}}=0$ and quantify it parametrically for the first time. As can be inferred from Eq. (1.1), in the presence of a primary source $Q_{\mathrm{B}}$, the $\mathrm{B} / \mathrm{C}$ ratio exhibits a plateau at high energies when the cross-section ratio $\sigma_{\mathrm{C} \rightarrow \mathrm{B}} /\left(\sigma^{\text {diff }}+\sigma_{\mathrm{B}}\right)$ becomes negligible with respect to the primary abundances ratio $N_{\mathrm{B}} / N_{\mathrm{C}}$. When adding a primary component of boron, the spectral index $\delta$ must increase to keep fitting the $\mathrm{B} / \mathrm{C}$ data at low energy, that is, here around $10 \mathrm{GeV} /$ nuc. This also implies that $D_{0}$ decreases with $N_{\mathrm{B}} / N_{\mathrm{C}}$ as a result of the anti-correlation between the diffusion parameters.

We have thus scanned the boron-to-carbon ratio at the source to study the variations of the best-fit values of $D_{0}$ and $\delta$ with respect to the reference model of Table 1. Our results are illustrated in Fig. 3, where the left panel features the confidence levels for $\delta$ (red) and $D_{0}$ (blue) as a function of the $N_{\mathrm{B}} / N_{\mathrm{C}}$ ratio. The $\mathrm{B} / \mathrm{C}$ fit is particularly sensitive to the last few AMS-02 points, notably the penultimate data point, around $214 \mathrm{GeV} /$ nuc, for which the $\mathrm{B} / \mathrm{C}$ ratio is found to be $\sim 9 \%$. In the right panel, the theoretical expectation for that point is plotted (solid red curve) as a function of the primary abundances ratio, while the dashed black curve indicates how the goodness of fit varies. It is interesting to note that a minor preference is shown for a non-vanishing fraction of primary boron, around $8 \%$, due to the marginal preference for a flattening of the ratio. The $N_{\mathrm{B}} / N_{\mathrm{C}}$ ratio is only loosely constrained to be below $13 \%$. Such a loose constraint would nominally mean that a spectral index $\delta$ more than three times larger than its benchmark value would be allowed, with a coefficient $D_{0}$ one order of magnitude smaller than indicated in Table 1. In fact, such changes are so extreme that they should probably be considered as unphysical to be in agreement with present acceleration schemes. The message is quite remarkable however. The degeneracy of the diffusion parameters with a possible admixture of primary boron is so strong that it dramatically degrades our capability of determining the best-fit values of $D_{0}$ and $\delta$, and beyond them the properties of turbulence, unless other priors are imposed.
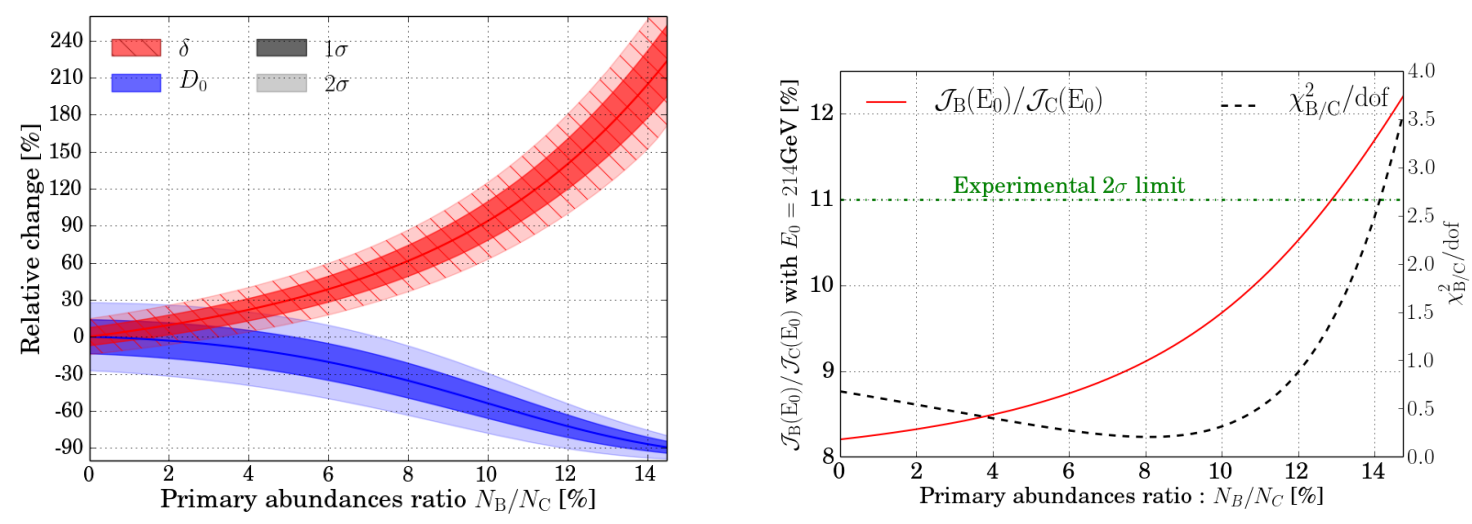

Figure 3: Left panel: variations of the best-fit propagation parameters $D_{0}$ (blue) and $\delta$ (red) relative to the benchmark values of Table 1, as a function of the primary boron-to-carbon injection ratio. The reference model corresponds to the conventional no boron hypothesis for which $N_{\mathrm{B}} / N_{\mathrm{C}}$ vanishes. Right panel: the theoretical value of the $\mathrm{B} / \mathrm{C}$ ratio at $214 \mathrm{GeV} / \mathrm{nuc}$ (solid red curve) is plotted as a function of the primary boron-to-carbon injection ratio. The dashed black curve indicates the goodness of the $\mathrm{B} / \mathrm{C}$ fit. As long as $N_{\mathrm{B}} / N_{\mathrm{C}}$ does not exceed $13 \%$, the theoretical $\mathrm{B} / \mathrm{C}$ ratio is within $2 \sigma$ from the AMS-02 measurement (dashed-dotted green curve). 


\section{Cross-section modeling}

The outcome of cosmic-ray propagation strongly depends on the values of the nuclear production $\sigma_{b \rightarrow a}$ and destruction $\sigma_{a}$ cross-sections with the ISM species, especially when we deal with secondary nuclei like boron. Since we consider here only the high-energy limit, we simply allowed for a rescaling of all the cross-sections. However, we distinguished between two cases: a correlated $\left(\nearrow \nearrow\right.$ ) or anti-correlated $\left(\nearrow \searrow\right.$ ) rescaling between the production $\sigma_{b \rightarrow a}$ and the destruction $\sigma_{a}$ cross-sections. Althought it worth noting that several relevant production cross-sections might be varied independently, since carbon, oxygen and nitrogen are in fact responsible for most of the produced boron.

First, we need to assess the reasonable range over which the various cross-sections of the problem are expected to vary. For this, we compared our reference models for the destruction and production cross-sections with those used in popular numerical propagation codes such as GALPROP [12] and DRAGON [13]. In this work we compare the values given directly by the default cross-section parameterisations without any renormalisation.

In the case of the destruction cross-sections $\sigma_{a}$, we compared our reference model [5] with the parameterisations of [14], [15] and [16]. The relative differences lets determine an indicative lower limit on the systematic uncertainties for the destruction cross-sections of roughly 2 to $10 \%$ for the $\mathrm{B} / \mathrm{C}$ ratio.

To gauge the uncertainties that affect the production cross-sections $\sigma_{b \rightarrow a}$, one can compare for example the semi-empirical approach proposed by [17], subsequently revised in 2000 and called here S\&T 00, and the parameterisation provided by [4] (hereafter Webber 03) as shown Fig. 4.

We present Fig. 4 only the anti-correlated $(\nearrow \searrow$ ) rescaling. The trend of the variations of $\delta$ and $D_{0}$ is well understood thanks to Eq. (1.2). From realistic assessments of the minimum systematic uncertainties of about $10 \%$ derived from the different cross-section models, we estimate a systematic uncertainty of $10 \%$ on $\delta$ and of $40 \%$ on $D_{0}$.

\section{CR propagation modeling}

A significant effort has been made in recent years to provide increasingly sophisticated modelling of the $\mathrm{CR}$ propagation environment. In the mere case of extracting CR propagation parameters from the $\mathrm{B} / \mathrm{C}$ ratio, our results leads to a surprising conclusion: these effect are of minor importance with respect to those presented above.

Geometric effect : The crude modelling of the diffusive halo as an infinite slab may appear too simplistic. We first estimate the effects of a 2D cylindrical diffusion box, modelled as in Fig. (1) right panel. The presence of a new escape surface at $R_{\mathrm{gal}} \simeq 20 \mathrm{kpc}$ is basically irrelevant: the bestfit $\delta$ and its error remain the same, with a statistically insignificant, $2 \%$ modification of the best-fit value of $D_{0}$. Secondly, we assess the effect of adding a radial dependence in the injection term according to [18], as opposed to the uniform hypothesis. This modification has minor effect since the best-fit $\delta$ remains almost unchanged and $D_{0}$ decrease by $13 \%$ within its one-sigma statistical uncertainty.

Convective wind: Although the high-energy CR propagation is mostly diffusive one can estimate the effect of adding advection outside the Galactic plane (for instance due to stellar winds). 

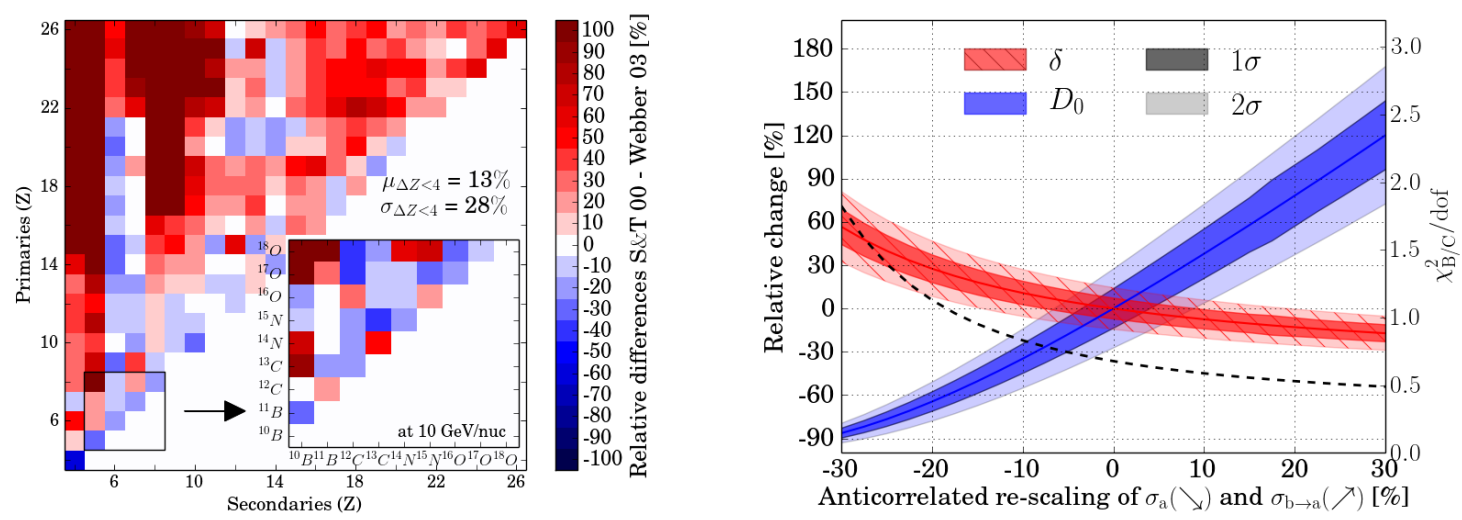

Figure 4: On the left panel: a 2D histogram feature the relative differences between two semi-empirical models currently used to calculate the production cross-sections $\sigma_{b \rightarrow a}$. Our reference model is Webber 03[4], and we compare it with S\&T 00 [17]. The charges of the parent and child nuclei are given on the vertical and horizontal axes, respectively. The relative difference in each bin is given by the arithmetic mean over the various isotopes of each element. A detailed view provides the most important channels for the B/C ratio studies. For a fragmentation of $\Delta Z<4$, we also give the first and second moments of the uncertainty distributions. On the right panel : effect of an anti-correlated rescaling of the nuclear cross-sections for boron production channels and destruction ones.

\begin{tabular}{lcccc}
\hline \hline & Wind & 1D/2D geometry & Cross-sections & Primary boron \\
\hline$\Delta D_{0} / D_{0}$ & $-40 \%$ & -2 to $-13 \%$ & $\pm 60 \%$ & 0 to $-90 \%$ \\
$\Delta \delta / \delta$ & $+15 \%$ & 0 to $+1 \%$ & $\pm 20 \%$ & 0 to $+100 \%$ \\
\hline
\end{tabular}

Table 1: Summary of the main systematics found in current analyses in determining the propagation parameters by fitting the $\mathrm{B} / \mathrm{C}$ ratio.

With the simplest model (constant velocity wind directed outside the galactic plane) and by neglecting adiabatic losses, we find that a variation of $15 \mathrm{~km} / \mathrm{s}$ in the velocity is roughly similar to a $1 \sigma$ shift in the benchmark parameters. Note, however, that the goodness of the fit worsens, or in other words, high-energy data are better described by a pure diffusive behaviour than by a convective-diffusive one.

\section{Conclusion}

Our main results are summarised in Table 1. We conclude that the Ansatz on the lack of primary injection of Boron represents the most serious bias, and requires multi-messenger studies to be addressed. If that uncertainty could be lifted, nuclear uncertainties would still represent a serious concern, which degrade the systematic error on the inferred parameters to the $20 \%$ level, or three times the estimated experimental sensitivity. In order to reduce this, a new nuclear crosssection measurement campaign is probably required. 


\section{References}

[1] Y. Genolini, A. Putze, P. Salati, and P. D. Serpico. Theoretical uncertainties in extracting cosmic-ray diffusion parameters: the boron-to-carbon ratio. ArXiv e-prints, April 2015.

[2] D. Maurin, F. Donato, R. Taillet, and P. Salati. Cosmic rays below $\mathrm{z}=30$ in a diffusion model: new constraints on propagation parameters. Astrophys.J., 555:585-596, 2001.

[3] Proc. 33rd Intern. Cosmic Ray Conf., editor. Precision Measurement of the Cosmic Ray Boron-to-Carbon Ratio with AMS [AMS collaboration], 2013.

[4] W. R. Webber, A. Soutoul, J. C. Kish, and J. M. Rockstroh. Updated Formula for Calculating Partial Cross Sections for Nuclear Reactions of Nuclei with $\mathrm{Z}=28$ and $\mathrm{E}>150 \mathrm{MeV} \mathrm{Nucleon}^{-1}$ in Hydrogen Targets. Apjs, 144:153-167, January 2003.

[5] R. K. Tripathi, F. A. Cucinotta, and J. W. Wilson. Universal Parameterization of Absorption Cross Sections. Technical report, January 1997.

[6] R. K. Tripathi, F. A. Cucinotta, and J. W. Wilson. Accurate universal parameterization of absorption cross sections III - light systems. Nuclear Instruments and Methods in Physics Research B, 155:349-356, September 1999.

[7] K. Lodders. Solar System Abundances and Condensation Temperatures of the Elements. Apj, 591:1220-1247, July 2003.

[8] D. Maurin, F. Melot, and R. Taillet. A database of charged cosmic rays. , 569:A32, September 2014.

[9] Pasquale Blasi. The origin of the positron excess in cosmic rays. Phys.Rev.Lett., 103:051104, 2009.

[10] Pasquale Blasi and Pasquale D. Serpico. High-energy antiprotons from old supernova remnants. Phys.Rev.Lett., 103:081103, 2009.

[11] P. Mertsch and S. Sarkar. AMS-02 data confront acceleration of cosmic ray secondaries in nearby sources. Prd, 90(6):061301, September 2014.

[12] A. W. Strong and I. V. Moskalenko. Models for galactic cosmic-ray propagation. Advances in Space Research, 27:717-726, 2001.

[13] C. Evoli, D. Gaggero, D. Grasso, and L. Maccione. Cosmic Ray propagation in the Galaxy and diffuse gamma-ray emission. In F. A. Aharonian, W. Hofmann, and F. Rieger, editors, American Institute of Physics Conference Series, volume 1085 of American Institute of Physics Conference Series, pages 380-383, December 2008.

[14] V S Barashenkov and A Polanski. Electronic guide for nuclear cross-sections: version 1994. Technical Report E2-94-417. JINR-E2-94-417, Joint Inst. Nucl. Res., Dubna, Oct 1994.

[15] J. R. Letaw, R. Silberberg, and C. H. Tsao. Proton-nucleus total inelastic cross sections - an empirical formula for E greater than $10 \mathrm{MeV}$. , 51:271-275, March 1983.

[16] H. P. Wellisch and D. Axen. Total reaction cross section calculations in proton-nucleus scattering. , 54:1329-1332, September 1996.

[17] R. Silberberg, C. H. Tsao, and A. F. Barghouty. Updated Partial Cross Sections of Proton-Nucleus Reactions. , 501:911-919, July 1998.

[18] I. Yusifov and I. Küçük. Revisiting the radial distribution of pulsars in the Galaxy. , 422:545-553, August 2004. 